\title{
Publisher's Note: Domain-width model for perpendicularly magnetized systems with Dzyaloshinskii-Moriya interaction [Phys. Rev. B 96, 144408 (2017)]
}

T. N. G. Meier, M. Kronseder, and C. H. Back

(Received 10 November 2017; published 16 November 2017)

DOI: 10.1103/PhysRevB.96.179902

This paper was published online on 6 October 2017 with incorrect text on page 1. On page 1, right-hand column, the first two sentences of the first paragraph should read as "Furthermore, the investigation of DMI was also extended to the stripe domain phase at zero field in these systems [24,25,28]. However, most domain models, except for [28], treat only the case of strong DMI for Co/Pt-based multilayer stacks, which forces the domain walls into the pure Néel wall state, and neglect the in-plane magnetostatic energy of the Néel walls [4,25]." The paper has been corrected as of 6 November 2017. The text is correct in the printed version of the journal. 